\title{
(Article)
}

\section{Virtual screening of selected phytochemicals from four antimalarial plants predicted potential inhibitors of wild type and mutant forms of Dihydrofolate Reductase-Thymidylate Synthase in Plasmodium sp.}

Moses N. Ikegbunam ${ }^{1,2}$, InnocentMary Ejiofor ${ }^{3}$, Valentine Onwuakpa ${ }^{1}$, Vincent C. Duru ${ }^{2,4}$, Akachukwu Amarachi ${ }^{2,5}$, Emmanual Oranu ${ }^{6}$, Jude Okoyeh ${ }^{7}$ and Samuel Egieyeh ${ }^{8}$

${ }^{1}$ Department of Pharmaceutical Microbiology and Biotechnology, Nnamdi Azikiwe University, Awka, Nigeria, mn.ikegbunam@unizik.edu.ng ${ }^{2}$ Molecular Research Foundation for Students and Scientists, Nnamdi Azikiwe University, Awka, Nigeria

${ }^{3}$ Department of Pharmacognosy and Traditional Medicine, Nnamdi Azikiwe University, Awka, Nigeria, ii.ejiofor@unizik.edu.ng

${ }^{4}$ Department of Parasitology and Entomology, Nnamdi Azikiwe University, Awka, Nigeria,vc.duru@unizik.edu.ng

5 Department of Pharmacology and Toxicology, Nnamdi Azikiwe University, Awka, Nigeria.ac.akachukwu@unizik.edu.ng

${ }^{6}$ Department of Pharmaceutical and Medicinal Chemistry, Chukwuemeka Odimegwu Ojukwu University, Igbariam, Nigeria. doctoremma10@gmail.com

${ }^{7}$ Department of Biology and Clinical Laboratory Science, School of Arts and Sciences, Neumann University, Aston. emekaok@hotmail.com

${ }^{8}$ Computational Pharmacology and Cheminformatics Group, School of Pharmacy, University of the Western Cape, Bellville 7535 Cape Town South Africa.segieyeh@uwc.ac.za

\section{Correspondence:}

Samuel Egieyeh

segieyeh@uwc.ac.za

$+27843477250$ 
Abstract: Resistance to Pyrimethamine, an antimalaria medicine has been reported to be due to mutations in Dihydrofolate reductase-thymidylate synthase (DHFR-TS). Phytochemicals, particularly from plants that been used in ethnomedicine, have been reported to have privileged structures that might bind strongly to the mutants of DHFR-TS. The aim of this study is to identify phytochemicals of Acalypha wilkesiana, Cymbopogon citratus, Azadirachta indica, and Morinda lucida with high binding affinities for the Plasmodium falciparum DHFR-TS. The threedimensional structures of the phytochemicals, wide type and mutant forms of DHTR-TS were obtained from PubChem and Protein Databank (PDB) respectively. They were appropriately prepared and molecular docking simulations was implemented to predict binding affinities of the phytochemicals to the wildtype and mutant forms of DHTR-TS. Druglikeness assessment was implemented to triage the top binding phytochemicals and molecular dynamics simulations was done to establish the stability of the interaction of the top-ranked phytochemical with one of the mutants of DHFR-TS. Nineteen phytochemicals showed higher binding affinities to both the wild type and mutant forms DHFR-TS than Pyrimethamine. Molecular dynamics revealed that the receptor-ligand binding of luteolin, the top-ranked, drug like phytochemicals, to the quadruple mutant was stable.

Keywords: Malaria, Resistance, Mutation, Virtual screening, Phytochemicals, Dihydrofolate Reductase - Tymidylate Synthase

\section{Introduction}


Malaria is a recognized health burden in the African and Asian regions of the world [1]. An estimate of 229 million cases of malaria that resulted in 409,000 deaths occurred worldwide in 2019, and the causative agent in the majority of these cases was Plasmodium falciparum [2]. Historically, a lot of strategies have been employed in an effort to eradicate malaria, including vector control, prophylaxis and treatment of already infected individuals by the use of chemical agents [3]. The above strategies, coupled with adequate funding and political commitment, have led to the eradication of malaria in some parts of the world [3]. Despite these efforts, malaria is still endemic in some parts of the world. The development and spread of Plasmodium falciparum resistance to inexpensive and previously effective antimalaria drugs, and the development of resistance by the vector, mosquito, to commonly used insecticides, pose serious threats to the eradication of malaria thereby increasing the mortalities and cases of treatment failures [4]. The development of resistance to the current first-line agent for the treatment of uncomplicated malaria (Artemisinin-base combination therapy, ACT) has also been reported [5].

Previously, antifolate drugs (including proguanil and pyrimethamine) which inhibit the dihydrofolate reductase - thymidylate synthase, were used in the chemotherapy and prophylaxis of Plasmodium falciparum malaria in sub-Saharan Africa [6]. However, the development of resistance to these drugs hindered their clinical uses [7]. It has been shown that mutation in the dihydrofolate reductase domain of the gene coding for their target enzyme, dihydrofolate reductase - thymidylate synthase (DHFR-TS), correlates with resistance by Plasmodium falciparum to these agents [8]. As the number of mutations increases, the level of resistance to antifolate-antimalaria drugs generally increase [8]. The double mutant (PDB ID: 1J3J) [19] and quadruple mutant (PDB ID: 3QG2) [20] forms of DHFR-TS have been reported in Plasmodium falciparum. The emergence and spread of Plasmodium falciparum with these reported mutations that are resistant 
to known antifolate antimalarial drugs continues unabated. There is a need to search for new and effective agents, especially from herbal plants, against these wild type and mutated targets in Plasmodium falciparum.

A large number of herbs and plants have been used in traditional medicine to treat malaria, especially in malaria-endemic communities. These plants, which include Azadirachta indica, Morinda Lucida, Acalypha wilkesiana and Cymbopogon citratus, have been screened in vitro and found to possess antimalarial activities $[9,10]$. Several studies have analyzed and reported the chemical constituents (phytochemicals) from these plants $[11,12,13,14]$. The phytochemicals in the extracts of these plants, screened for antimalarial activities in vitro and in vivo, possess privileged three-dimensional structures that probably interact with biomolecules in Plasmodium $s p$. to bring about the reported antimalarial activities. Virtual screening of these phytochemicals against specific biomolecules or enzymes, like the mutants of DHFR-TS in Plasmodium sp., may identify promising phytochemicals that might inhibit the mutated enzymes. Such phytochemicals might be used as a template to develop novel antimalarial lead compounds against this resistance Plasmodium sp.

In this study, we characterized the wild type, double mutant and quadruple mutant of the DHFR-TS enzyme to gain insight into the effect of the double and quadruple mutations on the protein properties of the enzyme. Secondly, we sought to find phytochemicals that may show greater binding energy to wild type, double mutant and quadruple mutant of DHFR-TS enzyme than Pyrimethamine. Therefore, we conducted virtual screening of reported phytochemicals from Azadirachta indica, Morinda Lucida, Acalypha wilkesiana and Cymbopogon citratus against wild type, double mutant and quadruple mutants DHFR-TS enzyme using molecular docking and molecular dynamics techniques 


\section{Results}

2.1. Three-dimensional structural alignments and physicochemical characterization of wild type, double mutant and quadruple mutant of DHFR-TS proteins

The goal here is to gain insight into the structural and physicochemical differences among the wild type, double mutant and quadruple mutant of DHFR-TS proteins. Firstly, the sequence alignment of the three protein was done in order to identify the points of mutation on these proteins and also to determine if the mutation points on these proteins are within the binding sites of the proteins. The multiple alignment of the protein FASTA sequence (Figure 1) showed the points of mutation for the double mutant; 1J3J (C59R and S108N) and quadruple mutant; 3QG2 $(\mathrm{N} 51 \mathrm{I}+\mathrm{C} 59 \mathrm{R}+\mathrm{S} 108 \mathrm{~N}+\mathrm{I} 164 \mathrm{~L})$ relative to the wild type; 3UM8. From Figures 2, which shows the amino acids residues present in the ligand binding site for the proteins. It was observed that one mutated residue in the binding site of $1 \mathrm{~J} 3 \mathrm{~J}(\mathrm{~S} 108 \mathrm{~N})$ and two mutated residues (S108N and I164L) in the binding sites of 3QG2 were involved in the interactions with pyrimethamine.

CLUSTAL $0(1.2 .4)$ multiple sequence alignment

3062_1 Chains 3UN8_1|Chains 1J3J_1|Chains

3Q62_1|Chains 3UN8_1|Chains 1J3]_1|Chains

3Q62_1|Chains 3UN8_1|Chains 1J3]_1|Chains

3062_1|Chains 3UN8_1|Chains 1J3J_1|Chains

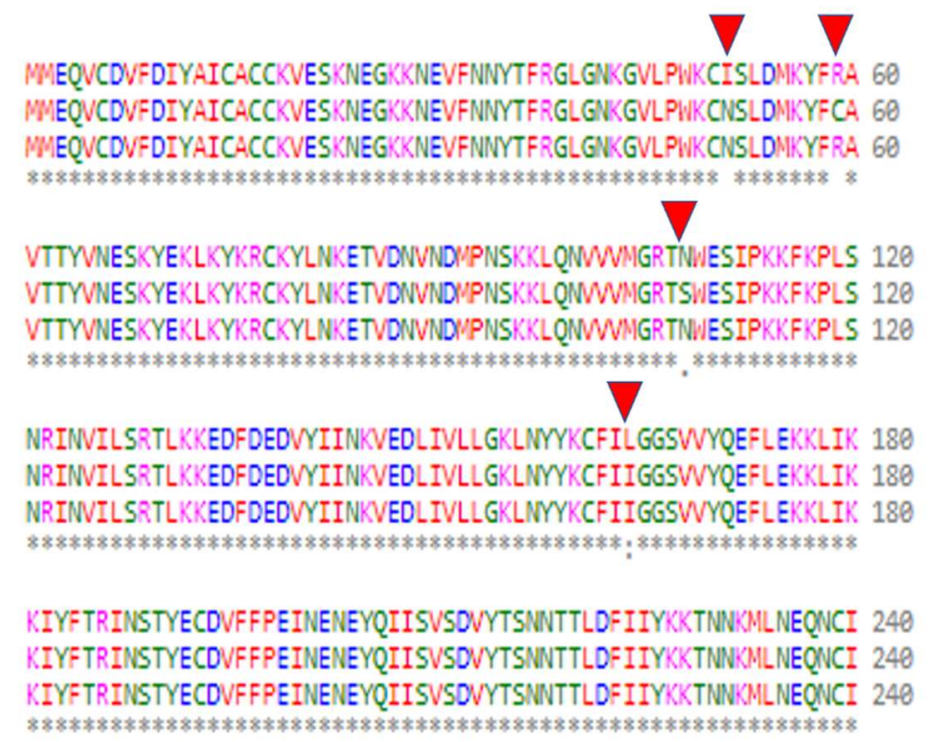


Figure 1: Multiple sequence alignment of the three proteins: wild-type (3UM8), double-mutant (1J3J) and quadruple mutant (3QG2) of DHFR-TS protein. Mutation points are highlighted with red arrows.
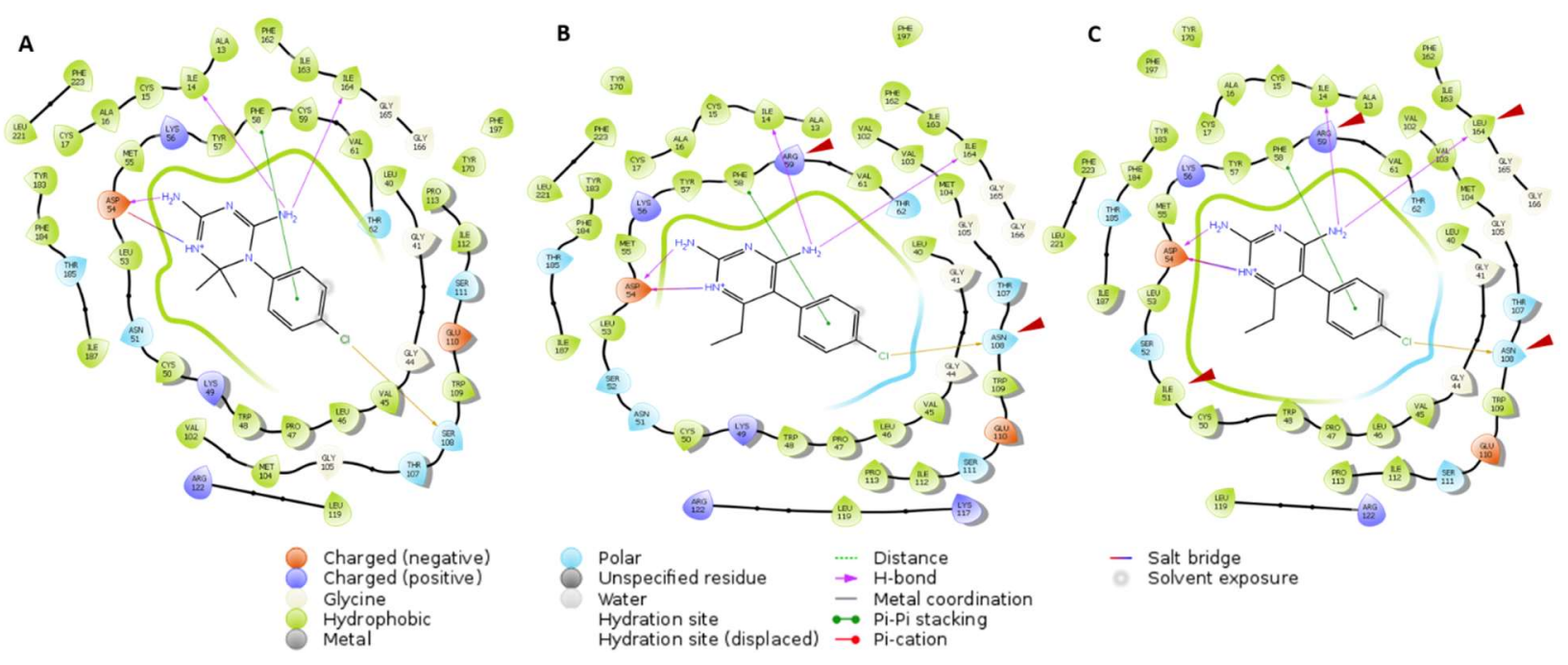

Figure 2: Amino acids present in the ligand binding site of wild-type (3UM8) (A), double-mutant (1J3J) (B) and quadruple mutant (3QG2) (C) of DHFR-TS protein. The red arrows highlight the mutated amino acid residues in the binding sites for1J3J (arginine 59 and asparagine 108) and 3QG2 (isoleucine 51, arginine 59, asparagine 108, and leucine 164)

Secondly, a superimposition of the three-dimensional models of double-mutant (1J3J) and the quadruple mutant (3QG2) on the wild-type (3UM8) was done to gain insight into possible effect of the point mutations on the three-dimensional models (Figure 3). The results showed a root mean standard deviation (RMSD) of $0.62 \AA$ between the three-dimensional models of the wild type and the double mutant of DHFR-TS protein, while the superimposition of the three-dimensional models of the wild type and quadruple mutant gave RMSD of 0.63 . The RMSD value of superimposed proteins gives the average deviation between the corresponding atoms of the threedimensional models of two proteins: the smaller the RMSD value, the more similar the two models [33]. The results show that the mutant proteins (double and quadruple) were to some extent structurally different from the wild type protein. The observed structural difference might be due to inherent protein flexibility and experimental resolution of the crystal structures. A closer look 
at the superimposed pyrimethamine binding site of the double mutant, quadruple mutant and wild type proteins shows slight deviation in the coordinates of a few residues at the site. Overall, this structural differences amongst the double mutant, quadruple mutant and wild type proteins, especially at the pyrimethamine binding site, might be a determinant of the mode and extent of interaction of the ligands with wild type and mutant DHFR-TS proteins.

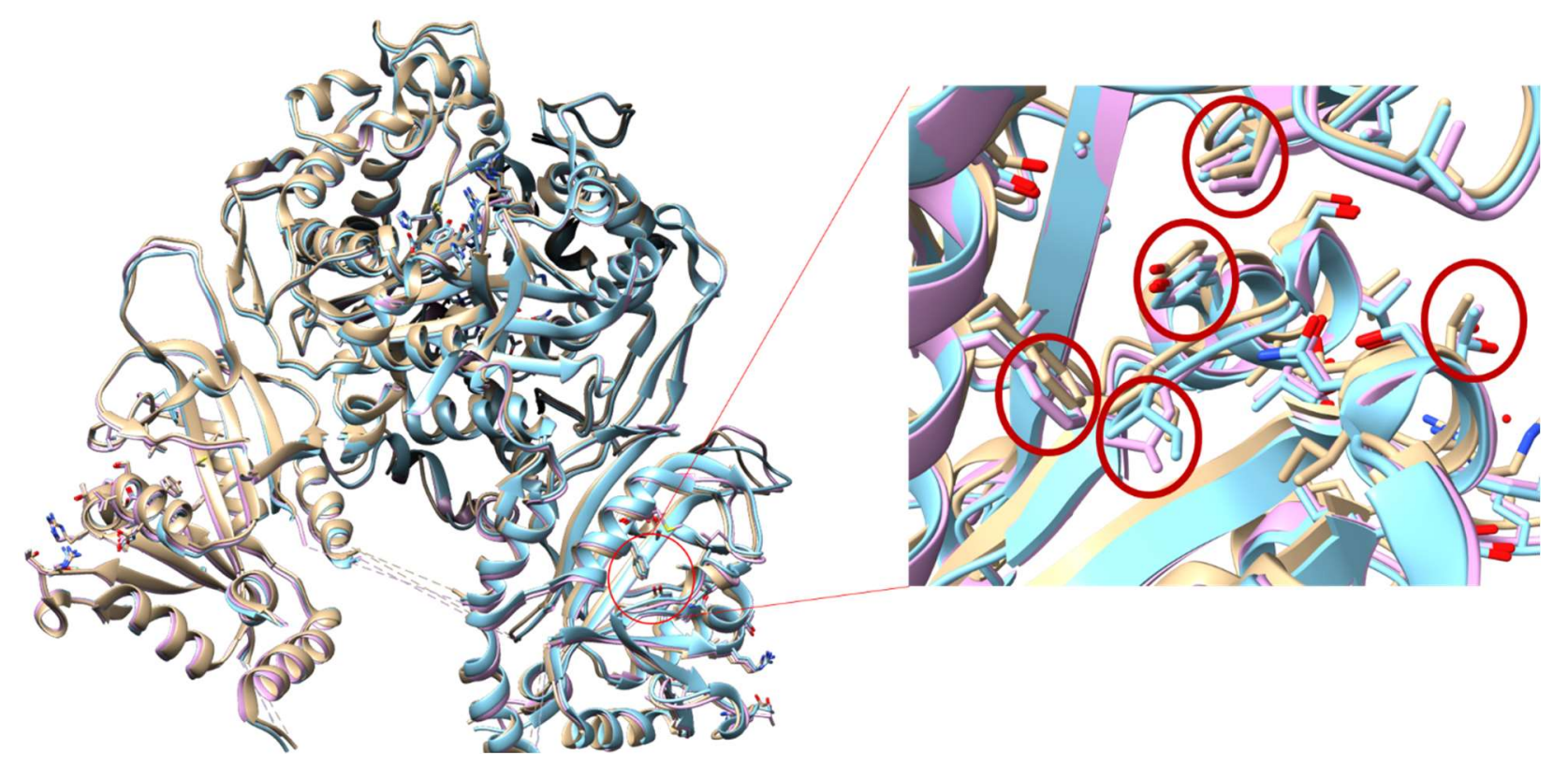

Figure 3: Superimposition of 1J3J (light blue) and 3QG2 (light purple) on 3UM8 (light brown). A closer view of the pyrimethamine binding site is also shown (a few residues with deviated coordinates are highlighted in red circle).

\subsection{Molecular surface characterization of wild type, double mutant and quadruple mutant of DHFR-TS protein}

The molecular surface characterization of the wild and mutant proteins was carried out to understand the effect of mutation on the surface properties of the proteins which has the ability to affect the inter protein interaction and intra protein interactions. The estimation of the electrostatic solvation energy on the macromolecular surfaces of the wild-type and mutated enzymes, obtained by solving the Poisson-Boltzmann (PB) equation, showed that the double mutant DHFR-TS had a 
significantly lower electrostatic solvation energy than the wild-type and quadruple mutant enzymes (Table 1). The electrostatic solvation energy of the quadruple mutant enzyme was slightly higher than the wild-type enzyme (Table 1). The results also showed differences in the distribution of the electrostatic solvation energy on the surface of the wild-type and mutant enzymes (Figure 4). A closer look at the molecular lipophilicity potential (MLP) of the pyrimethamine binding sites of the wild type and mutants showed significant difference in the distribution of the hydrophilicitylipophilicity surface (Figure 5). We further explored the polar and nonpolar surface domains on the wild-type and mutant enzymes of DHFR-TS. The results (Table 1) showed that the mutant DHFR-TS enzymes had reduced values for all the protein surface properties.

Table 1: Protein surface properties of the wild type (PDB:3UM8), double mutant B (PDB:1J3J) and quadruple mutant (PDB: 3QG2) proteins

\begin{tabular}{cccc}
\hline GENERAL PROTEIN PROPERTIES & 3UM8 & 1J3J & 3QG2 \\
\hline Mutations & & C59R+S108N & N51I+C59R+S108N+I164L \\
Electrostatic Solvation Energy (kcal/mol) & -15025.432 & -13607.013 & -15408.375 \\
Sum Positive Surface Area ( $\AA 2)$ & 26895.47 & 25003.55 & 25336.19 \\
Sum Negative Surface Area ( $\AA 2)$ & 15837.84 & 14939.08 & 15060.28 \\
Sum Donor Surface Area $(\AA 2)$ & 9007.72 & 8390.00 & 8415.34 \\
Sum Acceptor Surface Area ( $\AA 2)$ & 10044.40 & 9647.16 & 9660.91 \\
Hydrophobic moment & 659.04 & 360.81 & 449.63 \\
\hline
\end{tabular}

The solvation of proteins affects their physicochemical properties, particularly the interactions of proteins with ligands and other proteins through the surface domains. 

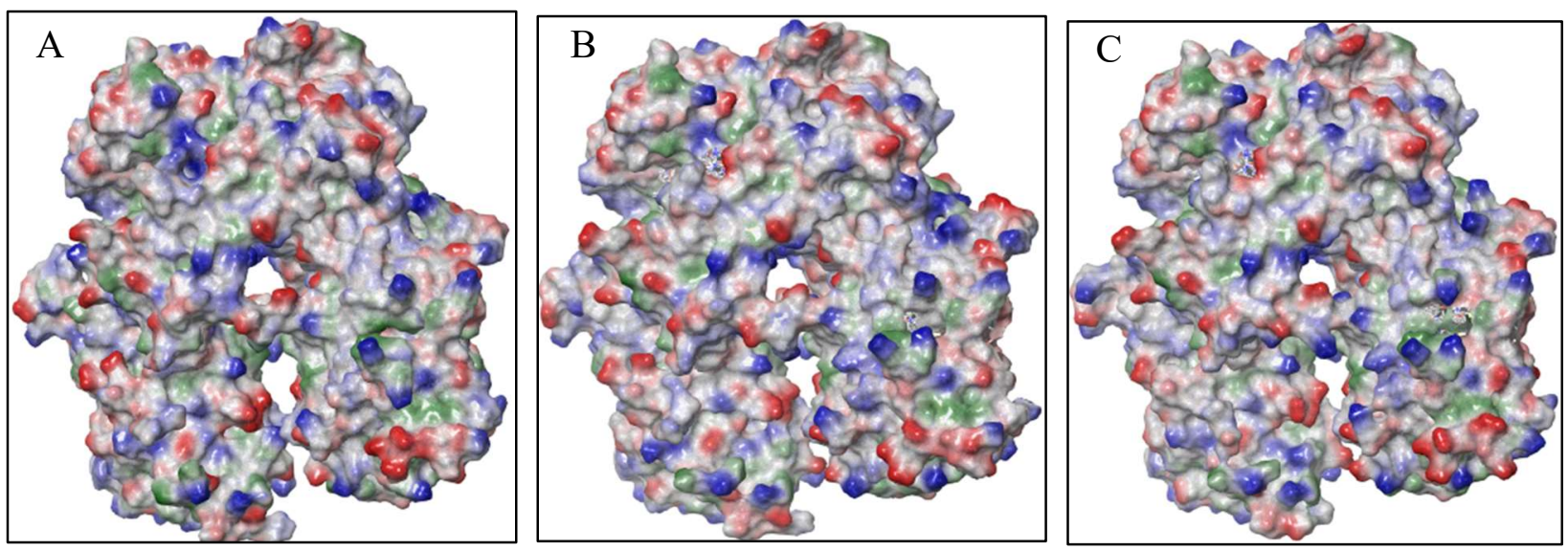

Figure 4: Molecular graphics views of the proteins; Wild type A (PDB:3UM8), double mutant B (PDB:1J3J) and quadruple mutant C (PDB: 3QG2) with surface electrostatic solvation potential distribution.

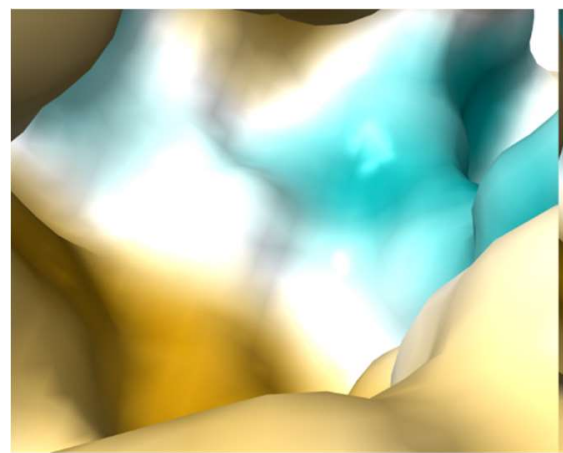

3UM8

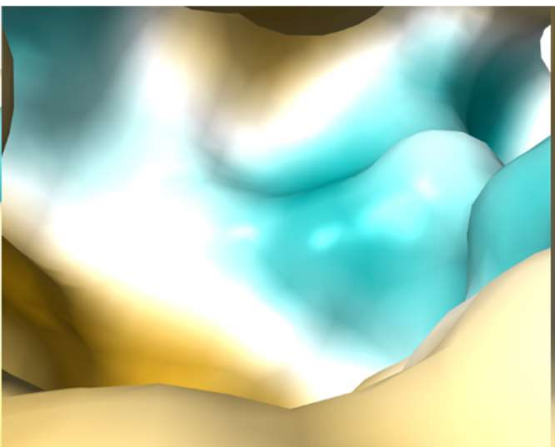

1J3J

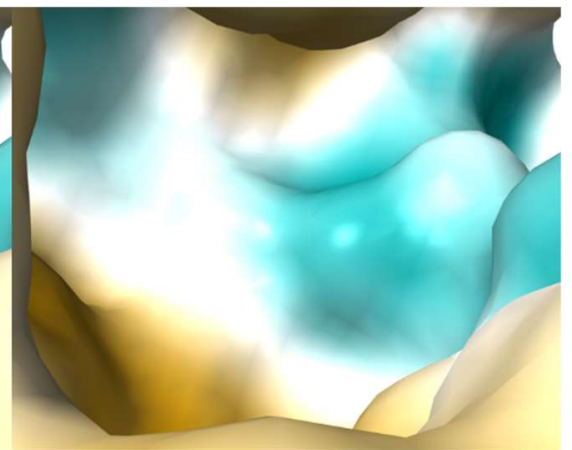

3QG2

Figure 5. Molecular lipophilicity potential (MLP) maps of the pyrimethamine binding site of the wild type (3UM8), double mutant (1J3J) and quadruple mutant (3QG2) of DHFR-TS protein. Colouring on the molecular surface range from dark cyan (most hydrophilic) to white to dark goldenrod (most lipophilic). A significant difference in the surface map was observed between the wild type (3UM8) and the mutants (1J3J and 3QG2). 
2.3. Molecular docking of the phytochemicals on wild type, double mutant and quadruple mutant of DHFR-TS enzyme

The molecular docking of the phytochemicals was performed on the three proteins in order to identify phytochemicals with better binding affinities than pyrimethamine, which was used as a reference compound, and the phytochemicals-proteins interaction pattern at the binding sites of these proteins. Phytochemicals with binding free energy less than that of pyrimethamine are presented in Table 2. Lower binding free energy suggest better ligand binding to the protein. The results show that lower number of phytochemicals had better binding free energies than pyrimethamine when docked on to the $1 \mathrm{~J} 3 \mathrm{~J}$ double mutant, while higher but almost equal number of phytochemicals had better binding free energies than pyrimethamine when docked on to the wildtype (3UM8) and the quadruple mutant (3QG2). The order of binding of the phytochemicals (binding profile) was distinctively different. For example, corilagin was predicted as the top binding phytochemical to the wildtype (3UM8) protein, protolimonoid was the top binding phytochemical to the double mutant $(1 \mathrm{~J} 3 \mathrm{~J})$ protein, while alpha-amyrin was the top binding phytochemical to the quadruple mutant (3QG2) protein. The structural diversity of these top binding phytochemicals (Figure 6) to these proteins further give credence to the difference in the ligand interaction potential of the three proteins due to the reported mutations. 
Table 2: Phytochemicals with binding free energies $((\mathrm{kcal} / \mathrm{mol})$ lower than pyrimethamine after the docking simulation. The average and standard deviation of binding free energies from three docking simulations for every compound are presented.

\begin{tabular}{|c|c|c|c|c|c|}
\hline \multicolumn{2}{|c|}{ 3UM8 } & \multicolumn{2}{|c|}{$1 \mathrm{~J} 3 \mathrm{~J}$} & \multicolumn{2}{|l|}{ 3QG2 } \\
\hline Phytochemicals & Binding affinity $( \pm \mathrm{SD})$ & Phytochemicals & Binding affinity $( \pm \mathrm{SD})$ & Phytochemicals & $\begin{array}{l}\text { Binding affinity } \\
( \pm \mathrm{SD})\end{array}$ \\
\hline Corilagin & $-11.13 \pm 0.05$ & Protolimonoid & $-11.18 \pm 1.45$ & alpha-amyrin & $-10.38 \pm 0.75$ \\
\hline Lupeol & $-11.00 \pm 0.00$ & Sesamolin & $-10.23 \pm 0.05$ & Scutellarin & $-9.88 \pm 0.13$ \\
\hline 17-hydroxyazadiradione & $-10.93 \pm 0.05$ & Scutellarin & $-9.90 \pm 0.00$ & Apigenin-7-O-glucoside & $-9.75 \pm 0.70$ \\
\hline alpha-amyrin & $-10.80 \pm 0.00$ & Apigenin-7-O-glucoside & $-9.70 \pm 0.20$ & Protolimonoid & $-9.63 \pm 1.00$ \\
\hline Azadiradione & $-10.80 \pm 0.00$ & Geraniin & $-9.60 \pm 0.00$ & Corilagin & $-9.50 \pm 0.00$ \\
\hline Stigmast-4-en-3-one & $-10.50 \pm 0.00$ & Stigmasterol & $-9.53 \pm 1.09$ & Luteolin & $-9.50 \pm 0.00$ \\
\hline Nimbocinol & $-10.50 \pm 0.00$ & Ellagic acid & $-9.50 \pm 0.00$ & Stigmasterol & $-9.33 \pm 0.73$ \\
\hline Protolimonoid & $-10.40 \pm 0.00$ & Punicalin & $-9.50 \pm 0.00$ & Quercetin & $-9.30 \pm 0.00$ \\
\hline Nimolinone & $-10.40 \pm 0.00$ & Naringenin & $-9.40 \pm 0.00$ & Apigenin & $-9.20 \pm 0.00$ \\
\hline Oleana-11-13(18)-diene & $-10.30 \pm 0.40$ & Quercetin & $-9.40 \pm 0.00$ & Geraniin & $-9.10 \pm 0.00$ \\
\hline Sesamolin & $-10.30 \pm 0.14$ & Cholesterol & $-9.38 \pm 0.05$ & Oleana-11-13(18)-diene & $-9.10 \pm 0.00$ \\
\hline Taraxerol & $-10.30 \pm 0.00$ & Apigenin & $-9.30 \pm 0.00$ & nimbocinol & $-9.03 \pm 0.68$ \\
\hline Rutin & $-10.23 \pm 0.05$ & Campesterol & $-9.30 \pm 1.81$ & Punicalin & $-9.00 \pm 0.00$ \\
\hline Stigmasterol & $-10.20 \pm 0.00$ & Brevifolincarboxylicacid & $-9.28 \pm 1.65$ & Dihydrocholesterol & $-8.98 \pm 0.65$ \\
\hline Azadirone & $-10.15 \pm 0.10$ & Luteolin & $-9.20 \pm 0.00$ & Azadirone & $-8.93 \pm 1.32$ \\
\hline Isonimocinolide & $-10.08 \pm 0.05$ & Dihydrocholesterol & $-9.10 \pm 0.00$ & Beta-amyrin & $-8.90 \pm 0.00$ \\
\hline Naheedin & $-10.08 \pm 0.05$ & Corilagin & $-9.00 \pm 0.00$ & Epicatechin & $-8.80 \pm 0.00$ \\
\hline Quadrangularic_acid & $-10.00 \pm 0.00$ & Icariin & $-9.00 \pm 0.00$ & Ginsenoside & $-8.80 \pm 0.00$ \\
\hline Scutellarin & $-10.00 \pm 0.00$ & Nimbocinol & $-9.00 \pm 0.00$ & Brevifolincarboxylicacid & $-8.80 \pm 0.80$ \\
\hline Nimolicinol & $-9.90 \pm 0.00$ & Pyrimethamine & $-9.00 \pm 0.00$ & Rosmarinic_acid & $-8.78 \pm 0.85$ \\
\hline Mahmoodin & $-9.80 \pm 0.00$ & & & 17-hydroxyazadiradione & $-8.70 \pm 0.00$ \\
\hline Gedunin & $-9.70 \pm 0.00$ & & & beta-sitosterol & $-8.68 \pm 0.26$ \\
\hline Methylbrevifolincarboxylate & $-9.60 \pm 0.00$ & & & Taraxerol & $-8.63 \pm 0.05$ \\
\hline Apigenin-7-O-glucoside & $-9.60 \pm 0.00$ & & & Azadiradione & $-8.60 \pm 0.00$ \\
\hline Nimbolide & $-9.43 \pm 0.05$ & & & Lupeol & $-8.60 \pm 0.00$ \\
\hline beta-sitosterol & $-9.40 \pm 0.00$ & & & nimolinone & $-8.53 \pm 0.49$ \\
\hline Cholesterol & $-9.40 \pm 0.00$ & & & Chlorogenic_acid & $-8.50 \pm 0.93$ \\
\hline Ginsenoside. & $-9.20 \pm 0.00$ & & & Mahmoodin & $-8.50 \pm 0.00$ \\
\hline Icariin & $-9.10 \pm 0.34$ & & & Quadrangularic_acid & $-8.48 \pm 0.65$ \\
\hline Campesterol & $-9.10 \pm 0.00$ & & & campesterol & $-8.40 \pm 1.07$ \\
\hline Dihydrocholesterol & $-9.00 \pm 0.00$ & & & cholesterol & $-8.40 \pm 1.23$ \\
\hline Beta-amyrin & $-9.00 \pm 0.00$ & & & Naringenin & $-8.40 \pm 0.12$ \\
\hline Chlorogenic_acid & $-8.78 \pm 0.05$ & & & Gedunin & $-8.35 \pm 0.10$ \\
\hline Ellagic_acid & $-8.60 \pm 0.00$ & & & isonimocinolide & $-8.30 \pm 0.00$ \\
\hline Luteolin & $-8.40 \pm 0.00$ & & & Methylbrevifolincarboxylate & $-8.30 \pm 0.14$ \\
\hline Apigenin & $-8.40 \pm 0.00$ & & & Ellagic_acid & $-8.25 \pm 0.97$ \\
\hline Epicatechin & $-8.40 \pm 0.00$ & & & Nimolicinol & $-8.20 \pm 0.00$ \\
\hline Naringenin & $-8.38 \pm 0.88$ & & & Nimbolide & $-8.18 \pm 0.26$ \\
\hline Quercetin & $-8.38 \pm 0.05$ & & & Rutin & $-8.08 \pm 0.75$ \\
\hline Brevifolincarboxylicacid & $-8.30 \pm 0.00$ & & & Naheedin & $-7.95 \pm 0.10$ \\
\hline Rosmarinic_acid & $-8.30 \pm 0.00$ & & & Stigmast-4-en-3-one & $-7.93 \pm 0.05$ \\
\hline Pyrimethamine & $-7.90 \pm 0.00$ & & & Icariin & $-7.85 \pm 0.10$ \\
\hline & & & & Pyrimethamine & $-7.80 \pm 0.20$ \\
\hline
\end{tabular}

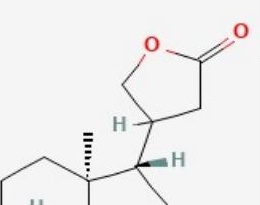

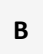

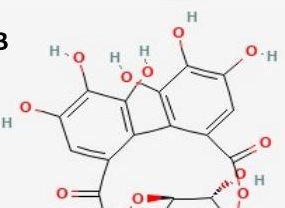

C 
Figure 6. The structural diversity of protolimonoid (A), corilagin (B) and alpha-amyrin (C) that are the top binding phytochemicals to the double mutant (1J3J) protein, wildtype (3UM8) protein and quadruple mutant (3QG2) protein respectively.

\subsection{Drug-likeness and toxicity filtration of the selected phytochemicals}

The drug-likeness filtration of the phytochemicals with binding free energies less than that of pyrimethamine was done based on Lipinski's rule of five to remove phytochemicals which have no druglike properties based on the Lipinski's rule. Phytochemicals with no Lipinski's rule violation are presented in Table 3. These phytochemicals with no Lipinski's rule violation (Table 3) were subjected to toxicity assessment using OSIRIS property explorer, to identify and filter out phytochemicals with either mutagenic, tumorigenic, irritant or reproductive effect. Phytochemicals without any of the toxicities are presented in Table 4 . Overall, just over $20 \%$ of the initially selected phytochemicals with good affinities for the three proteins were remaining after the druglikeness and toxicity filtration steps.

Table 3: Phytochemicals with no Lipinski violation 


\begin{tabular}{|c|c|c|c|c|c|}
\hline Phytochemicals & Binding affinity $( \pm \mathrm{SD})$ & Phytochemicals & Binding affinity $( \pm$ SD) & Phytochemicals & $\begin{array}{l}\text { Binding affinity } \\
( \pm \text { SD })\end{array}$ \\
\hline 17-hydroxyazadiradione & $-10.93 \pm 0.05$ & Sesamolin & $-10.23 \pm 0.05$ & Luteolin & $-9.50 \pm 0.00$ \\
\hline Azadiradione & $-10.80 \pm 0.00$ & Ellagic_acid & $-9.50 \pm 0.00$ & Quercetin & $-9.30 \pm 0.00$ \\
\hline Nimbocinol & $-10.50 \pm 0.00$ & Naringenin & $-9.40 \pm 0.00$ & nimbocinol & $-9.030 .68 \pm$ \\
\hline Sesamolin & $-10.30 \pm 0.14$ & Quercetin & $-9.40 \pm 0.00$ & Azadirone & $-8.93 \pm 1.32$ \\
\hline Azadirone & $-10.15 \pm 0.10$ & Brevifolincarboxylicacid & $-9.28 \pm 1.65$ & Epicatechin & $-8.80 \pm 0.00$ \\
\hline Isonimocinolide & $-10.08 \pm 0.05$ & Luteolin & $-9.20 \pm 0.00$ & Brevifolincarboxylicacid & $-8.80 \pm 0.80$ \\
\hline Quadrangularic_acid & $-10.00 \pm 0.00$ & Nimbocinol & $-9.00 \pm 0.00$ & Rosmarinic_acid & $-8.78 \pm 0.85$ \\
\hline Nimolicinol & $-9.90 \pm 0.00$ & Pyrimethamine & $-9.00 \pm 0.00$ & 17-hydroxyazadiradione & $-8.70 \pm 0.00$ \\
\hline Gedunin & $-9.70 \pm 0.00$ & & & Azadiradione & $-8.60 \pm 0.00$ \\
\hline Methylbrevifolincarboxylate & $-9.60 \pm 0.00$ & & & Chlorogenic_acid & $-8.50 \pm 0.93$ \\
\hline Nimbolide & $-9.43 \pm 0.05$ & & & Quadrangularic_acid & $-8.48 \pm 0.65$ \\
\hline Chlorogenic_acid & $-8.78 \pm 0.05$ & & & Naringenin & $-8.40 \pm 0.12$ \\
\hline Ellagic_acid & $-8.60 \pm 0.00$ & & & gedunin & $-8.35 \pm 0.10$ \\
\hline Luteolin & $-8.40 \pm 0.00$ & & & isonimocinolide & $-8.30 \pm 0.00$ \\
\hline Epicatechin & $-8.40 \pm 0.00$ & & & Methylbrevifolincarboxylate & $-8.30 \pm 0.14$ \\
\hline Naringenin & $-8.38 \pm 0.88$ & & & Ellagic_acid & $-8.25 \pm 0.97$ \\
\hline Quercetin & $-8.38 \pm 0.05$ & & & Nimolicinol & $-8.20 \pm 0.00$ \\
\hline Brevifolincarboxylicacid & $-8.30 \pm 0.00$ & & & Nimbolide & $-8.18 \pm 0.26$ \\
\hline Rosmarinic_acid & $-8.30 \pm 0.00$ & & & Pyrimethamine & $-7.80 \pm 0.20$ \\
\hline Pyrimethamine & $-7.90 \pm 0.00$ & & & & \\
\hline
\end{tabular}

Table 4: Phytochemicals with no toxicity violation on OSIRIS

\begin{tabular}{|c|c|c|c|c|c|}
\hline \multicolumn{2}{|c|}{ 3UM8 } & \multicolumn{2}{|c|}{$1 \mathrm{~J} 3 \mathrm{~J}$} & \multicolumn{2}{|c|}{ 3QG2 } \\
\hline Phytochemicals & Binding affinity $( \pm \mathrm{SD})$ & Phytochemicals & Binding affinity $( \pm \mathrm{SD})$ & Phytochemicals & Binding affinity $( \pm \mathrm{SD})$ \\
\hline 17-hydroxyazadiradione & $-10.93 \pm 0.05$ & Sesamolin & $-10.23 \pm 0.05$ & Luteolin & $-9.50 \pm 0.00$ \\
\hline Azadiradione & $-10.80 \pm 0.00$ & Naringenin & $-9.40 \pm 0.00$ & Nimbocinol & $-9.030 .68 \pm$ \\
\hline Nimbocinol & $-10.50 \pm 0.00$ & Luteolin & $-9.20 \pm 0.00$ & Azadirone & $-8.93 \pm 1.32$ \\
\hline Sesamolin & $-10.30 \pm 0.14$ & Nimbocinol & $-9.00 \pm 0.00$ & Epicatechin & $-8.80 \pm 0.00$ \\
\hline Azadirone & $-10.15 \pm 0.10$ & Pyrimethamine & $-9.00 \pm 0.00$ & Rosmarinic_acid & $-8.78 \pm 0.85$ \\
\hline Luteolin & $-8.40 \pm 0.00$ & & & 17-hydroxyazadiradione & $-8.70 \pm 0.00$ \\
\hline Epicatechin & $-8.40 \pm 0.00$ & & & Azadiradione & $-8.60 \pm 0.00$ \\
\hline Naringenin & $-8.38 \pm 0.88$ & & & Naringenin & $-8.40 \pm 0.12$ \\
\hline Rosmarinic_acid & $-8.30 \pm 0.00$ & & & Pyrimethamine & $-7.80 \pm 0.20$ \\
\hline Pyrimethamine & $-7.90 \pm 0.00$ & & & & \\
\hline
\end{tabular}

2.5. Phytochemicals with increased binding affinity to the quadruple mutant (3QG2) in comparison to the wild type (3UM8)

Presented in Table 5 are the phytochemicals with increased binding affinity (as expressed by the binding free energy) to quadruple mutant protein relative to the wildtype protein. The absolute difference in binding free energies, Lipinski's rule properties and total polar surface area (tPSA) values for these set of phytochemicals are also presented. From the absolute difference in 
binding free energies, it was observed that luteolin might have the greatest binding affinity for the quadruple mutant protein relative to the wildtype. Since the goal here is to find the compound that showed good potential to bind to both the wildtype and the quadruple mutant protein, especially binding to the quadruple mutant, luteolin became the obvious choice. The dynamics of the interaction of luteolin with the quadruple mutant protein was examined with molecular dynamic simulation and analysis.

Table 5: Phytochemicals with increased binding free energy against the quadruple mutant (3QG2) in comparison to the wild type (3UM8) showing the difference in binding free energy, Lipinski's rule of five properties and total polar surface area (tPSA) values

\begin{tabular}{ccccccccc}
\hline Phytochemicals & 3UM8 & 3QG2 & $\begin{array}{c}\text { Absolute } \\
\text { difference } \\
\text { in binding } \\
\text { free } \\
\text { energies }\end{array}$ & MW & LogP & HBD & HBA & tPSA \\
\hline Luteolin & $-8.40 \pm 0.00$ & $-9.50 \pm 0.00$ & 1.10 & 286.24 & 1.97 & 4 & 6 & 111.12 \\
Epicatechin & $-8.40 \pm 0.00$ & $-8.80 \pm 0.00$ & 0.40 & 290.27 & 1.37 & 5 & 6 & 110.37 \\
Rosmarinic_acid & $-8.30 \pm 0.00$ & $-8.78 \pm 0.85$ & 0.48 & 360.32 & 1.63 & 5 & 8 & 144.52 \\
Naringenin & $-8.38 \pm 0.88$ & $-8.40 \pm 0.12$ & 0.02 & 272.26 & 2.12 & 3 & 5 & 86.99 \\
Pyrimethamine & $-7.90 \pm 0.00$ & $-7.80 \pm 0.20$ & 0.10 & 248.72 & 2.84 & 4 & 4 & 77.83 \\
\hline
\end{tabular}

2.6. Visualisation of the top three phytochemicals frontrunners in DHFR-TS Quadruple mutant's (3QG2) binding site

The top three frontrunner phytochemicals in DHFR-TS Quadruple mutant's binding site were visualised using ligplot. The visualisation included observation of the binding interactions between the ligands and the receptor (Hydrogen and hydrophobic bonding), the binding site and the points of mutation. Pyrimethamine has interactions with two mutation points, one hydrogen bond interaction (Asn108) and hydrophobic interaction (Leu164), as shown in Figure 7A. Luteolin has hydrogen bond interaction with the residue at Asn108, which is one of the points of mutation, as shown in Figure 7B. Naringenin showed interactions with two mutation points, one hydrogen 
interaction with Asn108 and hydrophobic interaction with Leu 64 (Figure 7C), while Epicatechin has two hydrogen bond interactions with two mutation points, Asn108 and Leu164, (Figure 7D). From these observations, it could be suggested that interactions with the points of mutations affected the binding affinities of these ligands on the quadruple mutant enzyme in comparison to the wild-type enzyme. Calculation of the percentage increase in binding affinities of these phytochemicals from the wild-type to quadruple mutant revealed that luteolin had a $13.1 \%$ increase, Epicatechin $4.8 \%$, Rosmarinic acid $5.7 \%$, Naringenin $0.2 \%$, while Pyrimethamine had $-1.3 \%$ increment.

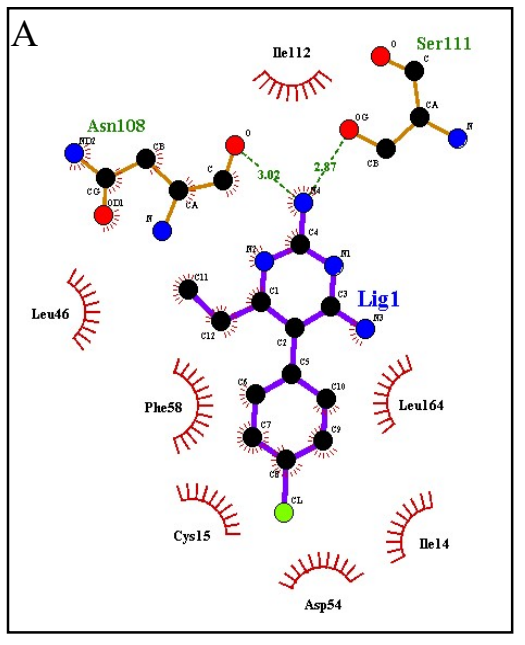

$\mathrm{C}$

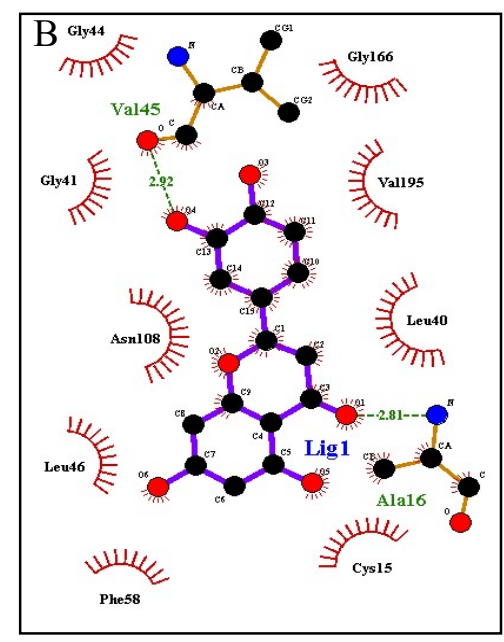

$\mathrm{D}$ 

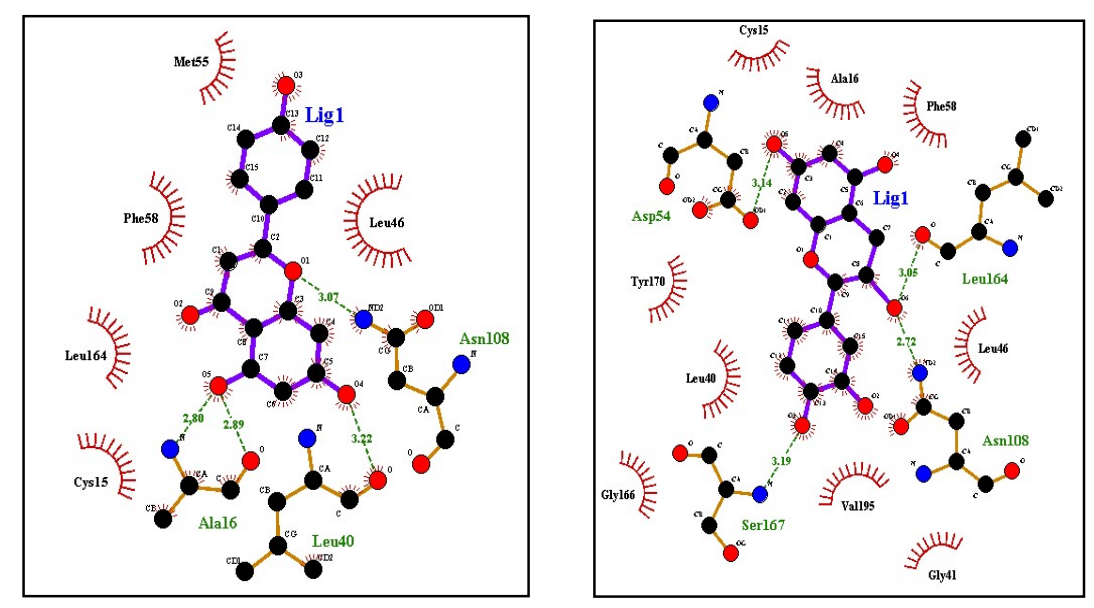

Figure 7. Ligplot representations of Pyrimethamine-quadruple mutant complex (A), Luteolinquadruple mutant complex (B), Naringenin-quadruple mutant (C) and Epicatechin-quadruple mutant (D)

\subsection{Receptor-ligand complex pharmacophore modelling}

The interaction of luteolin with the wildtype (3UM8), double mutant (1J2J) and quadruple mutant (3QG2) of DHFR-TS enzyme's binding site were visualised using Maestro's ligand interaction viewer. The visualisation showed diverse binding interactions between the luteolin and the wildtype (3UM8) (A), double-mutant (1J3J) (B) and quadruple mutant (3QG2) (C) of DHFR-TS enzymes (as previously observed for pyrimethamine in Figure 2). 


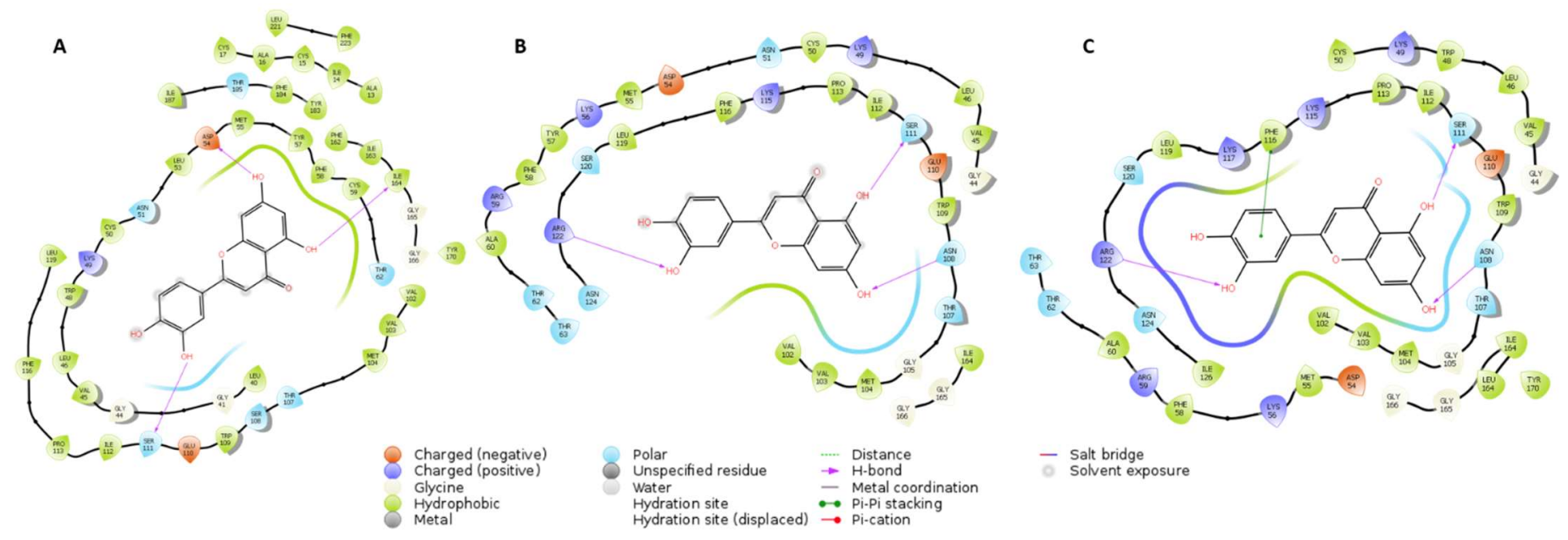

Figure 8. Interaction of luteolin with the amino acid residues present in the ligand binding site of wild-type (3UM8) (A), double-mutant (1J3J) (B) and quadruple mutant (3QG2) (C) of DHFR-TS protein. Luteolin showed interaction with asparagine 108 in the double-mutant (1J3J) (B) and quadruple mutant (3QG2) (C) of DHFR-TS protein.

Based on this observation, we developed the receptor-ligand complex pharmacophore models for luteolin and pyrimethamine complexed with the wild-type (3UM8) (A), double-mutant (1J3J) (B) and quadruple mutant (3QG2) (C) of DHFR-TS protein using PHASE in Maestro (Schrodinger $\left.{ }^{\circledR}\right)$. Figure 9 shows the receptor-ligand complex pharmacophore models of luteolin and pyrimethamine with the wild-type (3UM8) (A), double-mutant (1J3J) (B) and quadruple mutant (3QG2) (C) of DHFR-TS protein. From the models, it was observed the interaction of pyrimethamine with the wildtype DHFR-TS protein was facilitated by one aromatic ring, two hydrogen bond donors, and one hydrophobic entity. In contrast, interaction of pyrimethamine with the double mutant was facilitated by two aromatic ring systems and three hydrogen bond donors while the interaction with the quadruple mutant was facilitated by two aromatic ring systems and two hydrogen bond donors. Luteolin showed a different interaction, in comparison to pyrimethamine, with the wildtype DHFR-TS protein facilitated by two aromatic rings and two hydrogen bond donors. In contrast to what was observed for pyrimethamine, luteolin showed similar pharmacophore models in its interaction with the double and quadruple mutant of the DHFR-TS protein, facilitated by 
more entities including two aromatic rings, one hydrogen bond donor and three hydrogen bond acceptors.

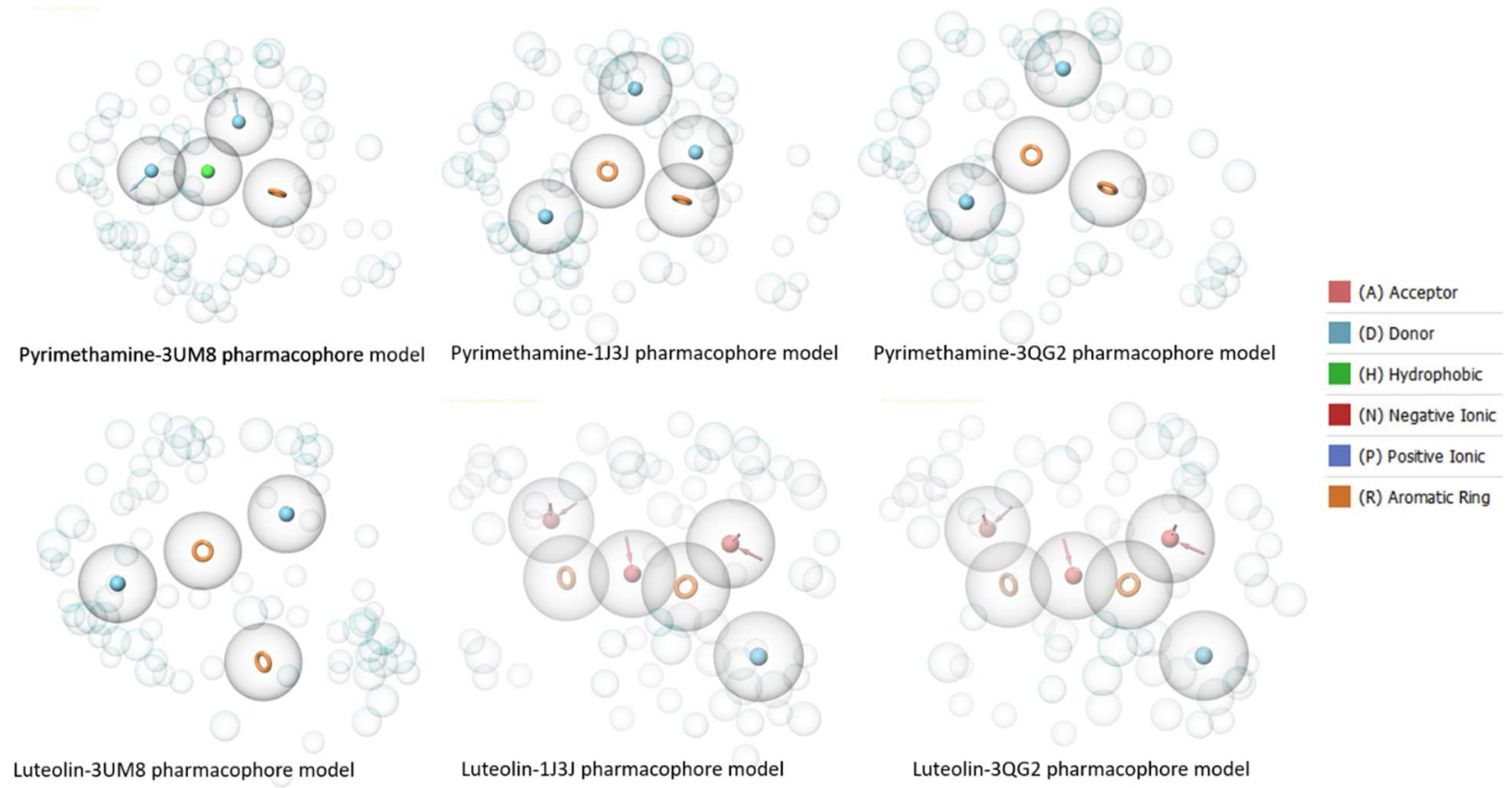

Figure 9. The receptor-ligand complex pharmacophore model of pyrimethamine (top panel) and luteolin (bottom panel) with the wildtype, double mutant and quadruple mutant of DHFR-TS protein.

\subsection{Protein-Ligand complex molecular dynamics simulation}

Molecular dynamics (MD) simulation of the phytochemical-protein interaction (luteolin3QG2 complex and pyrimethamine-3QG2 complex) was done to study the movement of the atoms in the protein over a period of time. The simulation captures a wide variety of important biomolecular processes, including conformational change, ligand binding, and protein folding, revealing the positions of all the atoms. These help to predict how biomolecules will respond at an atomic level to alterations such as mutation, phosphorylation, protonation, or the addition or removal of a ligand. 
The RMSD time series for the protein reveals that the protein was stable thermally with the highest and lowest RMSD values of 0.5 and $0.9 \AA$, respectively, as shown in figures $12 \mathrm{~A}, 12 \mathrm{~B}$ and $12 \mathrm{C}$, while the luteolin-mutant complex gave the highest and lowest RMSD values of 0.5 and 0.9 respectively as shown in figure $12 \mathrm{~A}$. Pyrimethamine also gave similar RMSD values as seen in figure $12 \mathrm{~B}$ and also observed in the overlapping of both luteolin-mutant and pyrimethaminemutant complexes RMSD presented in figure 12C. With the aid of the Root Mean Square Fluctuation (RMSF), which helps in the understanding of the region of the protein that undergoes fluctuation during molecular dynamic simulations, the flexibility of each of the residues of the protein can be calculated to get a better insight on to what extent the binding of the ligand affects the protein flexibility. The protein, protein-pyrimethamine complex and protein-luteolin complex had similar flexibility as can be observed in figure 13, even though the protein-pyrimethamine complex had fluctuation above $1.0 \AA$ at residues Cys50 and Leu81, while the protein-luteolin complex had fluctuation above 1.0Å at residues Asn24 and Ser52. Comparing mutant alone with mutant-luteolin and mutant-pyrimethamine complexes, it can be observed that the complexes fluctuated, as can be observed in figure 13, due to flexibility induced by the complex formation. Both complexes gave close level flexibility when compared with each other. 

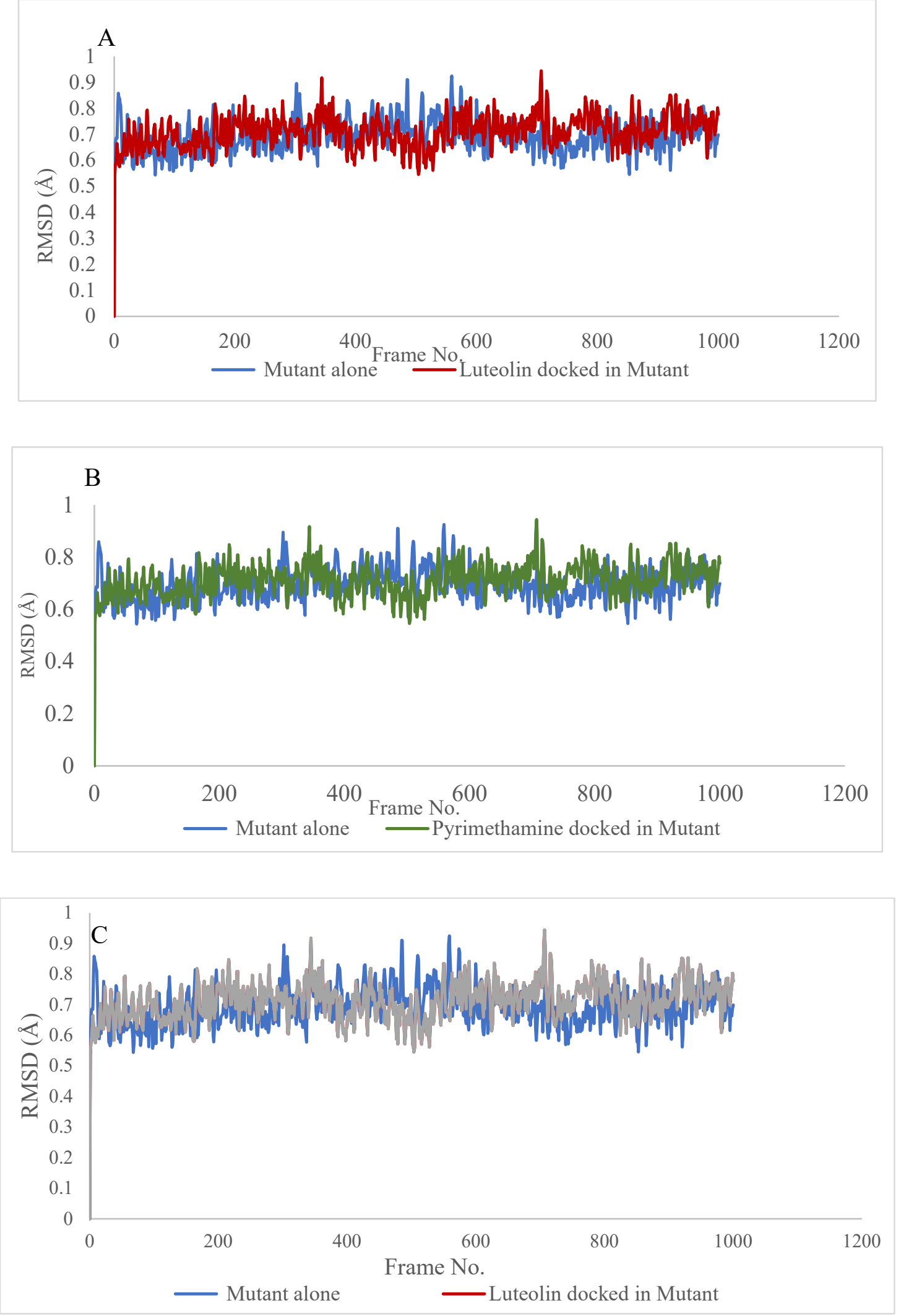

Figure 10: Root mean square deviation (RMSD) timeseries of Plasmodium falciparum target: (A) RMSD of free DHFR-TS qradruple mutant receptor and ligands (pyrimethamine and luteolin) bound complexes (B) RMSD of free DHFR-TS qradruple mutant receptor and ligand (luteolin)bound complex. (C) RMSD of free DHFR-TS qradruple mutant receptor and ligand (Pyrimethamine)bound complex 


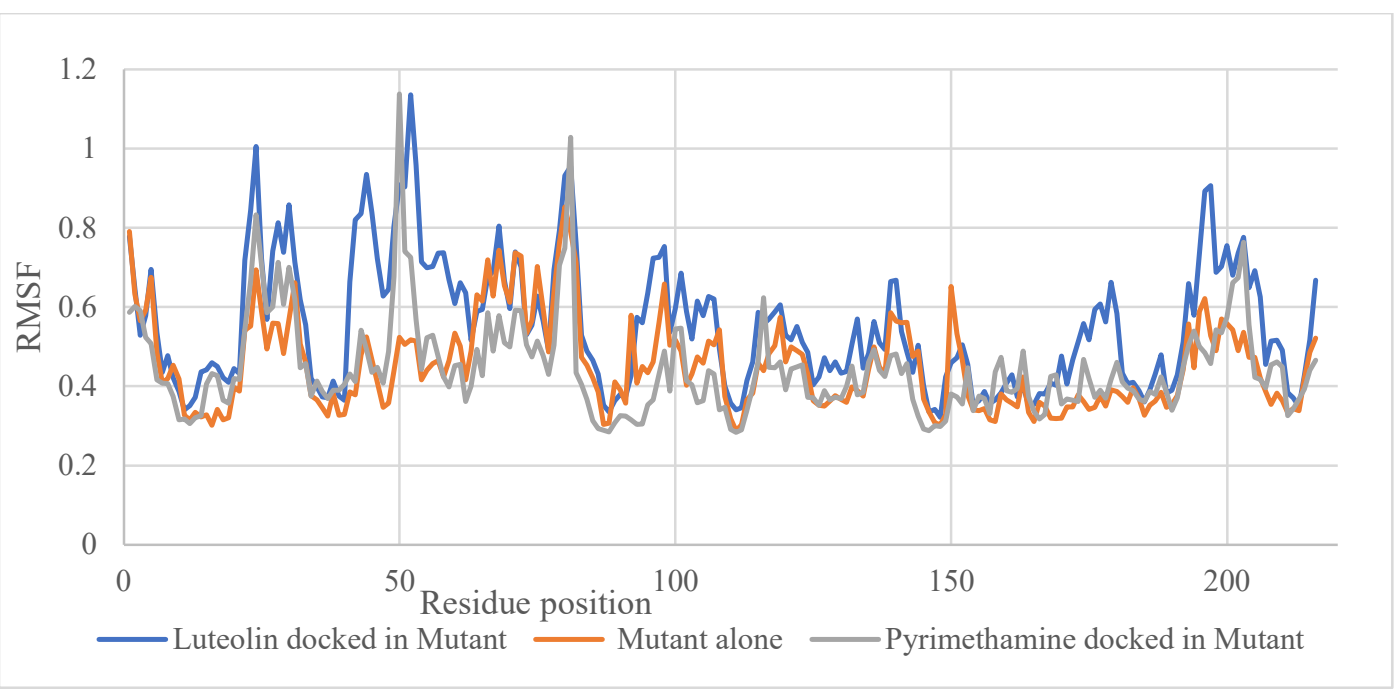

Figures 11: Residue-wise Root Mean Squared Fluctuations (RMSF) of mutant alone, luteolin docked in mutant and pyrimethamine docked in mutant.

PCA can be utilized in examining the relationship between various conformations sampled during the trajectory and is implemented in the Bio3D functions. Applying PCA to both distributions of experimental structures and molecular dynamics trajectories will be covered in detail in other vignettes. Their corresponding eigenvalue characterizes the percentage of the total mean square displacement (or variance) of atom positional fluctuations captured in each dimension.

For the PCA of the mutant alone, the first three principal components are responsible for $21 \%$ of the total variance, as seen in the eigenvalue rank plot. The first principal component (PC1) accounts for $9 \%$ of the variance, as shown in figure 14A. For the PCA of the mutant-luteolin complex, the first three principal components are responsible for $38.3 \%$ of the total variance, as seen in the eigenvalue rank plot. The first principal component (PC1) accounts for $19.9 \%$ of the 
variance, as shown in figure 14B. For the PCA of the mutant alone, the first three principal components are responsible for $19.3 \%$ of the total variance, as seen in the eigenvalue rank plot. The first principal component (PC1) accounts for $7.9 \%$ of the variance, as shown in figure $14 \mathrm{C}$
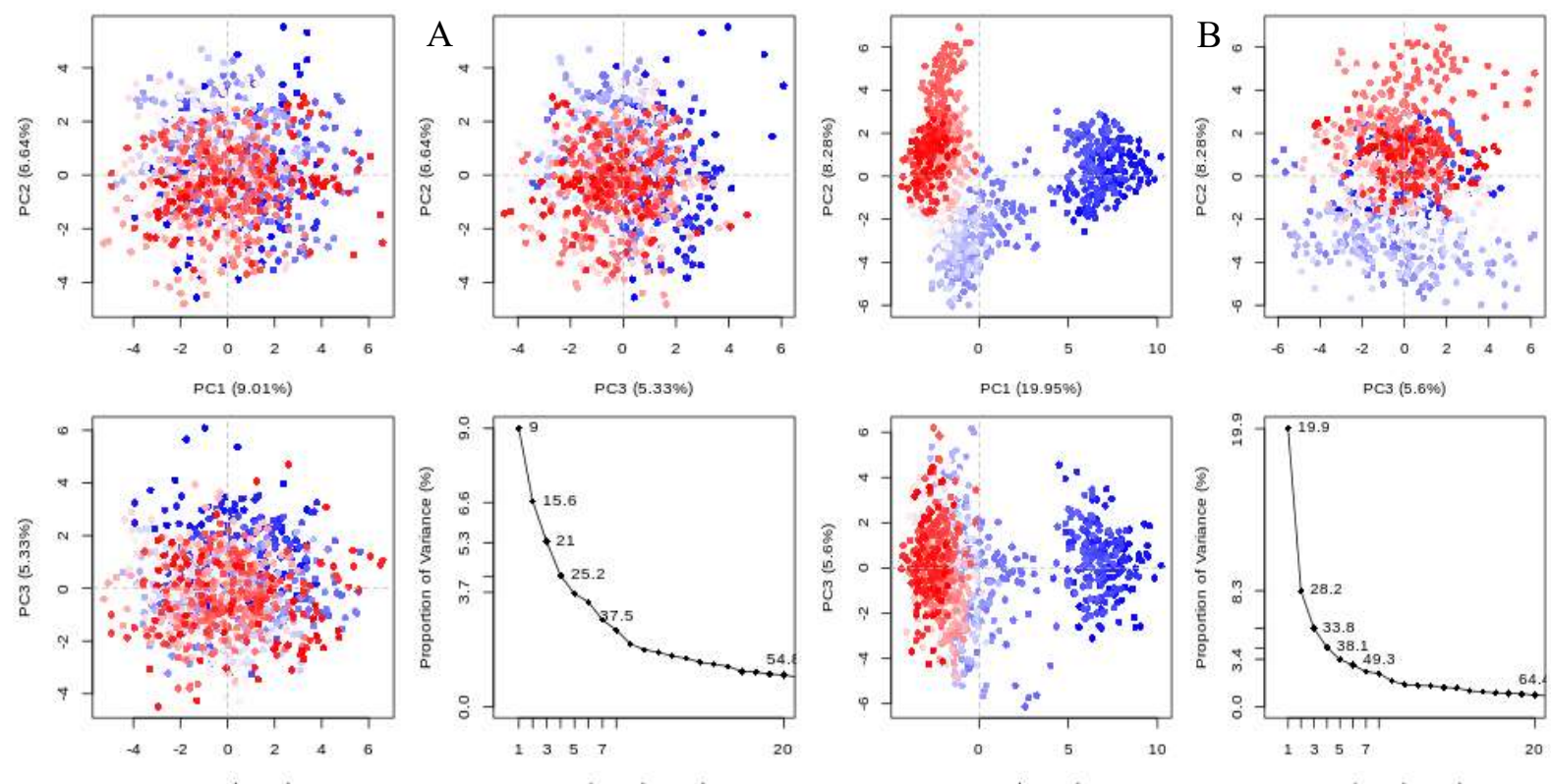

PC1

Eigenvalue Rank
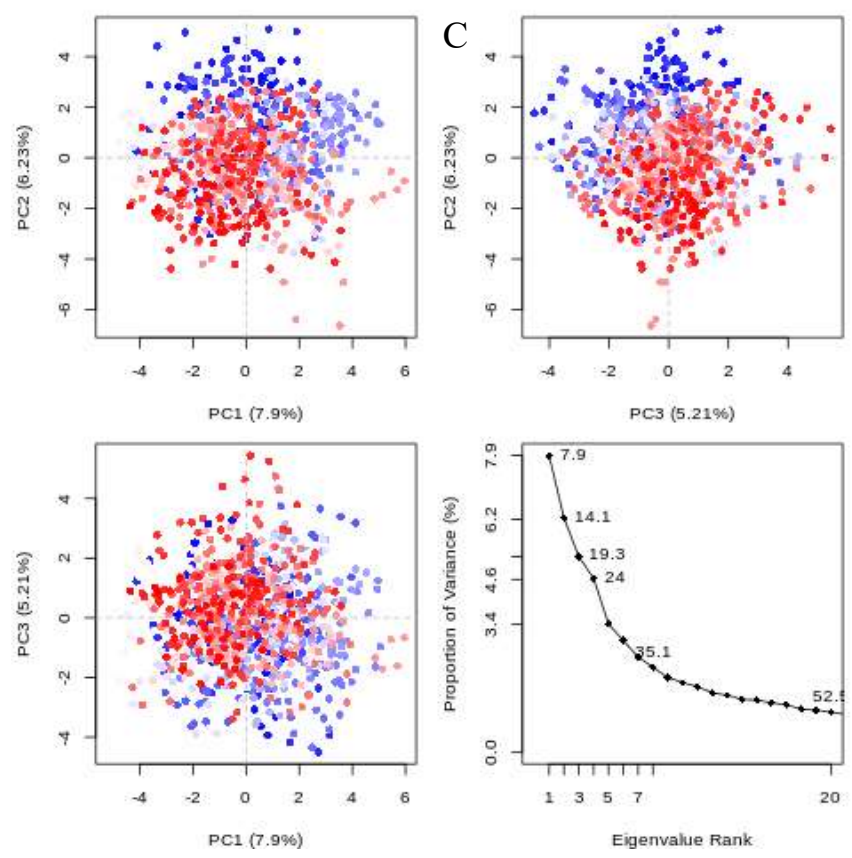

Figure 12: PCA results which include graphs of PC2 vs PC1, PC2 vs PC3, PC3 vs PC1 colored from blue to red in order of time, and an eigenvalue rank plot. A for mutant alone, $\mathrm{B}$ for mutant-luteolin complex and $\mathrm{C}$ for mutant-pyrimethamine complex. 


\section{Discussion}

The study set out to determine the binding affinities of reported phytochemicals in Acalypha wilkesiana, Cymbopogon citratus, Azadirachta indica, and Morinda lucida to the wild type and mutant forms of Plasmodium falciparum Dihydrofolate Reductase-Thymidylate synthase (DHFR-TS) in comparison with the reference compound, Pyrimethamine, using in silico molecular docking and stimulation.

Computer-aided drug design or in-silico approach in drug discovery and design has become an essential tool in modern days research. The huge cost of drug discovery and development and the length of time required has made the course of new drug development a challenging one. With components of computer-aided drug design like molecular docking, molecular dynamics, QSAR and ADMET tool and their reliable predictions, the process of drug discovery and development is accelerated.

In order to understand the effects of mutation on the surface properties of the proteins which has the ability to affect the inter protein interaction and intra protein interactions. The molecular surface characterization of the wild and mutant proteins (DHFR-TS) was carried out. The results suggest that there will be differences in the interaction potential of the wild-type and mutant enzymes of DHFR-TS due to the mutations. This might explain the observed differential (probably reduced) interaction of the mutant DHFR-TS with the inhibitor pyrimethamine, which resulted in the reported clinical failure of the drug in resistant Plasmodium falciparum.

Through molecular docking, the binding modes between a ligand and a protein can be predicted. On the other hand, for thousands of ages from early man, medicines and medicinal agents have been sourced from nature, mostly plants. Most of the medicines used today are isolated 
or developed from isolates obtained from natural sources. Most of these currently used medicines are developed from natural sources based on their use in traditional medicinal practices. In this study, 201 phytochemicals isolated from Acalypha wilkesiana, Cymbopogon citratus, Azadirachta indica, and Morinda lucida were docked against wild type, double mutant and quadruple mutant Dihydrofolate reductase-thymidylate synthase of Plasmodium falciparum. Among them, forty-one phytochemicals were selected for the wild type, ninety-one for the double mutant and forty-two for the quadruple mutant based on the best binding energies, lower than Pyrimethamine, our benchmark compound. These phytochemicals were assessed for Lipsinski's rule conformation and violation. Lipinski's rule states that, in general, an orally active drug has no more than one violation of the following criteria: No more than 5 hydrogen bond donors (the total number of nitrogenhydrogen and oxygen-hydrogen bonds), no more than 10 hydrogen bond acceptors (all nitrogen or oxygen atoms), molecular mass less than 500 daltons and octanol-water partition coefficient (log P) that does not exceed 5. Nineteen phytochemicals from wild type group, eight from the double mutant and eighteen from the quadruple mutant group conformed to Lipinski's rule. These phytochemicals were subjected to Toxicity Risk Assessment on Osiris Property Explorer platform for mutagenicity, tumorigenicity, irritating effects and reproductive effects. The assessment process relies on a precomputed set of structural fragments that give rise to toxicity alerts in case they are encountered in the structures uploaded. These fragment lists were created by rigorously shredding all compounds of the Registry of Toxic Effects of Chemical Substances (RTECS) database known to be active in a certain toxicity class. During the shredding, any molecule was first cut at every rotatable bond leading to a set of core fragments. These, in turn, were used to reconstruct all possible bigger fragments being a substructure of the original molecule. Afterwards, a substructure search process determined the occurrence frequency of any fragment (core and 
constructed fragments) within all compounds of that toxicity class. It also determined these fragment's frequencies within the structures of more than 3000 traded drugs. Based on the assumption that traded drugs are largely free of toxic effects, any fragment was considered a risk factor if it often occurred as a substructure of harmful compounds but never or rarely in traded drugs. Nine phytochemicals from the wild type group, five from double mutant and eight from the quadruple mutant, were predicted to have no form of toxicity. These phytochemicals contain no fragment or fragments known to have any of the toxicities listed according to the Registry of Toxic Effects of Chemical substances. Among these Phytochemicals, four showed increased binding affinities from the wild type to double mutant and to quadruple mutant, as shown in table 5. These changes in binding affinities could be attributed to the difference in the interaction pattern of these phytochemicals with the binding sites the mutated proteins.

To further gain insight into the interaction patterns of the phytochemicals and the reference drug with the wildtype and mutated protein, we constructed receptor-ligand complex pharmacophore models for luteolin (the top-rated phytochemical) and pyrimethamine. The pharmacophore models obtained encoded the three-dimensional organization of the required interaction pattern of the luteolin and pyrimethamine with the wildtype and mutant proteins (Figure 9). The increased entities and similar interaction pharmacophore models to the double and quadruple mutant proteins by luteolin suggest that the mutations did not affect the interaction of this phytochemical. This attests to the potential of luteolin to be effective against the mutated proteins.

Molecular dynamics simulations were undertaken for the quadruple mutant, pyrimethamine-quadruple mutant complex and luteolin-quadruple mutant complex. The two complexes showed a low level of RMSD values which indicate the stability of the complexes. The 
Root Mean Square Fluctuation (RMSF) of the mutant alone and the complexes the stability of the complexes, with the protein-luteolin complex showing more stability.

The dynamics of the system were also assessed using a two-dimensional projection of Principal Component Analysis for the prediction of motions in the complexes. PC1 denotes the most significant, and it accounts for the maximum variability in terms of internal motion of proteins, while PC2 accounts for the remaining variability. From the 2D projection, a complex with a stable cluster occupying less phase space represents a stable complex, while a less stable complex shows a non-stable cluster occupying more space. From the result, the quadruple mutantluteolin complex demonstrated a high level of stability.

\section{Materials and Methods}

\subsection{Creation of database of phytochemicals isolated from the target plants}

A database of 201 phytochemicals isolated from Acalypha wilkesiana, Cymbopogon citratus, Azadirachta indica, and Morinda Lucida was created through literature search.

4.2. Sequence and structure alignments of wild type, double mutant and quadruple mutant of DHFR-TS enzyme

Multiple sequence alignments of the three proteins used in the study was carried on Multiple Sequence Alignment Clustal Omega tool. The FASTA sequences of the proteins were obtain from Protein Data Bank and copied directly to the Clustal Omega platform [15]. Structure based multiple sequence alignment was done after superposition. The USCF chimera was used to conduct a structural comparison analysis (using the Needleman-Wunsch algorithm and BLOSUM62 matrix in USCF Chimera) of each mutant to the wildtype and estimate the root mean square deviation (RMSD).

\subsection{Characterization of wild type, double mutant and quadruple mutant of DHFR-TS enzyme}


The solvation energy of the wild type, 3UM8, double mutant, 1J3J and quadruple mutant, 3QG2 forms of Plasmodium falciparum DHFR-TS was calculated with PBEQ solver on CHARMM GUI. PBEQ Solver calculates and visualizes the electrostatic potential and solvation energy of molecules by solving the Poisson-Boltzmann (PB) equation.[16] BioLuminate ${ }^{\circledR}$ from Schrödinger [17] was used to further gain insight into the surface properties of the threedimensional structures of the wild-type and mutants of Plasmodium falciparum DHFR-TS.

\subsection{Preparation of proteins for molecular docking}

The structural data for the wild type, 3UM8 [18], double mutant, 1J3J [19] and quadruple mutant, 3QG2 [20] forms of Plasmodium falciparum DHFR-TS were obtained from the PDB database in PDB file format. The protein structures were prepared for molecular docking using UCSF chimera 1.11.2 software and Autodock tools 1.5.6.[21,22] Residues were edited using UCSF chimera 1.11.2; polar hydrogen and Kollman charges were added using Autodock tools 1.5.6.[22] Grid boxes of sizes and centres as shown in table 1 were marked around the

pyrimethamine binding pocket at $1.0 \AA$. The resulting structures were saved as pdbqt (Protein Data Bank, Partial Charge (Q), and Atom Type (T)) file ready for molecular docking. These receptor preparations were carried out as previously reported. [23]

\subsection{Preparation of ligands (Plant chemical structures)}

The 3D structures of the compiled 201 ligands from literature, including the reference compound, Pyrimethamine, were extracted from the Pubchem database [24] in SDF file format and converted to MOL2 file using Open Babel 2.4.1. The phytochemicals were subjected to 1000 steps of steepest descent and 100 steps of conjugate gradient energy minimization at a step size of 0.02 on UCSF Chimera-1.9. They were prepared for molecular docking simulations using 
MGLTools-1.5.6. [25, 26] Polar hydrogen bonds were, and Kollman charges were added, torsions and rotatable bonds were allowed to stay rotatable. Output was then generated as a pdbqt file.

\subsection{Validation of docking protocol}

In order to validate the molecular docking simulations protocol for the 3QG2, 3UM8 and 1J3J proteins, the PDB structure of these proteins in complex with Pyrimethamine, cycloguanil and Pyrimethamine respectively, were reproduced insilico. The deletion of the reference compounds from the proteins was done using UCSF Chimers-1.9.[27] Polar hydrogen, Kollman charges, grid box sizes and centers at grid space of $1.0 \AA$ were determined with MGLTools1.5.6. $[25,26]$ The reference compounds were subjected to 1000 steps of steepest descent and 100 steps of conjugate gradient energy minimization at a step size of 0.02 on UCSF Chimera-1.9. They were prepared for molecular docking simulations using MGLTools-1.5.6. Polar hydrogen bonds and Kollman charges were added, torsions and all rotatable bonds were allowed to stay rotatable. Output was then generated as a pdbqt file extension.

Molecular docking simulations were implemented locally using AutoDockVina ${ }^{\circledR}[28]$ on a Linux platform using the centers and sizes with a virtual screening shell script. Docked conformations were visualized in PyMol-1.4.1, and poses were compared with the experimental crystal structures of the reference compound.

4.7. Molecular docking of the phytochemicals on wild type, double mutant and quadruple mutant of DHFR-TS enzyme

The Phytochemicals were batched for molecular docking simulations against 3UM8, 1J3J and 3QG2 using virtual screening scripts. Molecular docking simulations were carried out in four replicates on a Linux platform using AutoDockVina ${ }^{\circledR}[28]$ and associated tools after validation of 
docking protocols. Binding free energy values $(\mathrm{kcal} / \mathrm{mol} \pm \mathrm{SD})$ were ranked in order to identify the frontrunner phytochemicals.

Assessment of druglikeness

The drug-likeness of the phytochemicals was evaluated using DruLito (Drug Likeness Tool) software. [29] Lipinski's rule five, which is considered as the rule of the thumb was utilized to filter the phytochemicals based on their drug-likeness characteristics. [30] The phytochemicals that had no Lipinski violation were subjected to in-silico toxicity analysis using the OSIRIS Property Explorer. [31] Phytochemicals with toxicity violations were delisted.

Receptor-ligand complex pharmacophore modeling

Receptor-ligand complex pharmacophore models were generated for luteolin and pyrimethamine complexed with the wild-type (3UM8) (A), double-mutant (1J3J) (B) and quadruple mutant (3QG2) (C) of DHFR-TS protein using PHASE in Maestro (Schrodinger®). Auto Epharmacophore method was used, hypothesis was set with maximum number of features to be generated at 7 , minimum feature-feature distance at 2.00, minimum feature-feature distance for feature of the same type at 4.00 and donors as vectors.

\subsection{Protein-Ligand complex molecular dynamics simulation}

The Plasmodium falciparum DHFR-TS quadruple mutant -ligand complex of Pyrimethamine and the selected top phytochemical, Luteolin, as obtained from the molecular docking step, were subjected to molecular dynamics simulation to understand the effect of their binding on the structural stability and conformational flexibility of DHFR-TS quadruple mutant. The Plasmodium falciparum DHFR-TS quadruple mutant enzyme with and without a docked 
ligand (Pyrimethamine with a binding free energy of $-7.90 \mathrm{kcal} / \mathrm{mol}$ and Luteolin with a binding free energy of $-9.50 \mathrm{kcal} / \mathrm{mol}$ ) were subjected to molecular dynamics simulations utilising the Simulation module in Molecular Operating Environment MOE 2019.01 (Molecular Operating Environment (MOE) 2019.01). The DHFR-TS receptor and DHFR-TS receptor-ligand complex was at different instances protonated and energy minimized with the MMFF94x force field to get the stable conformer of the protein complexes in a vacuum (molecular system). The molecular system was parameterized with Optimized Potentials for Liquid Simulations (OPLS-aa) forcefield, suitable for proteins and small organic molecules. Molecular dynamics simulations were done in three steps. We first heated the molecular system to $310 \mathrm{~K}$ (37 0C). Then an equilibration step was used to equilibrate the molecule system at $310 \mathrm{~K}(370 \mathrm{C})$ for 10 nanoseconds. Next, the simulation step was used to generate the trajectory of the molecular system at $310 \mathrm{~K}$ using the Nose-PoincareAndersen (NPA) algorithm for 100 nanoseconds (time step of each simulation was set to 0.02 picoseconds). Visualizations and data analysis were performed with VMD software and Bio3D on Galaxy Europe platform. [32]

\section{Conclusion}

Luteolin, Epicatechin, Rosmarinic acid and Naringenin showed better binding free energy, no Lipinski rules violation and no toxicity. Quadruple mutant DHFR-TS was chosen for molecular dynamics simulation because it is the dominant mutant. From the results obtained, RMSD and RMSF results reveal that the DHFR-TS-luteolin complex has relative high stability as compared to the DHFR-TS-pyrimethamine complex. Our study has identified phytochemicals present in Acalypha wilkesiana, Cymbopogon citratus, Azadirachta indica, and Morinda lucida responsible for their antimalarial properties using molecular docking methodologies and molecular dynamic 
simulations. Further in-vitro studies are needed to validate the results obtained from these in-silico studies.

Author Contributions: Conceptualization: IM and OV; methodology: EI, IM, AA and ES; software: ES; validation: ES; formal analysis: OE; writing — original draft preparation: IM,EI, OJ and ES; writing — review and editing: VD, OJ and AA; visualization: ES, OV, and ; supervision: IM and ES. All authors have read and agreed to the published version of this manuscript.

Funding: This research received no external funding.

Conflicts of Interests: The authors declare no conflicts of interests.

\section{REFERENCES}

1. Whitty, C.; Anasah, E. Malaria control stalls in high incidence areas: we have a fight on our hand, to regain lost momentum. Br Med J 2019, 365, 1756 - 1833.

2. World Health Organisation. World Malaria Report., Geneva 2020, License CCBYNCSA 3.0 IGO.

3. Mendis K, Rietveld A, Warsame M, Bosman, A.; Greenwood, B.; Wernsdorferet W. H. From malaria control to eradication: The WHO Perspective. Trop Med \& Int Health 2009, 14, 803-809.

4. Zheng, L.; Kafatos, F. The Anopheles mosquito: Genomics and transformation. In Wahlgren, M. and Perlmana, p. (Eds), Malaria: Molecular and Clinical Aspects, Singapore: Taylor and Francis E-library 2017, 117

5. Straimer, J.; Gnadig, N.; Amaratunga, C.; Witkowski , B.; Duru, V.; Pramundita, A.R.; Dacheux, M.; Khim, N.; Zheng, L.; Lam S.; Gregory P.D.; Urnov, F.D.; MercereauPuijalon, O.; Benoit-Vical, F.; Fairhurst R.M.; Ménard, D.; Fidock, D.A. K13_propeller mutation confer artemisin resistance in Plasmodium falciparum clinical isolates. Science 2015, 34, 6220, 428-431.

6. Basco, L.; Ringwald, P.; Molecular epidemiology of malaria in Yaounde, Cameroon, VI: Sequence variations in the Plasmodium falciparum dihydrofolate - Thymidylate synthase. gene and in vitro resistance to pyrimethamine and cycloguanil. Am J Trop Med Hyg, 2000, 62, 2, 271-276. 
7. World Health Organisation. World Mal Rep., Geneva 2016, License: CCBY-NC-SA 3.0 IGO.

8. Karmchumwongpaisan, S.; Quarrell, R.; Charoensetakul, N.; Ponsinet, R.; Vilaivan, T.; Vanichtanankul, J.; Tarnchompoo, B.; Sirawaraporn, W.; Lowe, G.; Yuthavong, Y. Inhibitors of multiple mutants of Plasmodium falciparum dihydrofolate reductase and their antimalarial activities. $J$ Med Chem 2004, 47, 673-680.

9. Johnson-Ajinwo, O.; Antimalarial activation of Acalypha wilkisiana, J Pharm Care Health Sys. 2015, 2.

10. Farahna, M.; Bedri, S.; Khalid S.; Idris, M.; Pillai, C.R.; Khalil, E.A. Anti-plasmodial effects of Azadirachta Indica in experiments cerebral malaria: Apoptosis of cerebellar purkinje cells of mice as a marker. N Am J Med Sc 2010, 2, 11, 518-525.

11. Igwe, K.; Madubuike, A.; Otuokere, I, et al. Studies on the medicinal plant Acalypha wilkisian ethanol extract Phytocomponents by GCMS analysis. Global J Sc Front Res 2016, 16, 2, 49-55.

12. Onyeike, E.; Ikweuchi, J.; Ikewuchi, C. Quantitative high-performance liquid chromatography analysis of simple terpenes, carotenoid, phytosterols and flavonoids in the leaves of Acalypha wilkesiana Muell arg. Pac J Sc Tech 2015, 11, 2, 480-487.

13. Ntoga, P.; Baldolvini, N.; Mouray, E.; Mambu, L.; Belong, P.; Grellier, P. Activity of Ocimum basilicum, Ocimum canum, and Cymbopogon citratus essential oil against Plasmodium falciparum and mature-stage larvae of Anopheles fonestis S.S. Parasite 2004, 21,31 .

14. El-Hawary, S.; El-tantawy, M.; Rabeh, M.; Badr, W.K. Chemical composition and biological activities of essential oils of Azadirachta indica A Juss. Int J Appl Res Nat Prod 2103, 6, 4, 33-42.

15. Sievers, F.; Wilm, A.; Dineen, D.; Gibson, T.J.; Karplus, K.; Li, W.; Lopez, R.; McWilliam, H.; Remmert, M.; Söding, J.; Thompson, J.D.; Higgins, D.G. Fast, Scalable Generation of High-Quality Protein Multiple Sequence Alignments Using Clustal Omega. Molecular Systems Biology 2011, 7, 539.

16. Jo, S.; Vargyas, M.; Vasko-Szedlar, J.; Roux, B.; Im, W. PBEQ-Solver for online visualization of electrostatic potential of biomolecules, Nucleic Acids Research 2008, 36, 2, W270-W275. 
17. Schrödinger Release 2021-1: BioLuminate, Schrödinger, LLC, New York, NY, 2021.

18. Vanichtanankul, J.; Taweechai, S.; Uttamapinant, C.; Chitnumsub, P.; Vilaivan, T.; Yuthavong, Y.; Kamchonwongpaisan, S. Combined Spatial Limitation around Residues 16 and 108 of Plasmodium falciparum Dihydrofolate Reductase Explains Resistance to Cycloguanil. Antimicrob. Agents Chemother 2012, 56, 3928-3935

19. Yuvaniyama, J.; Chitnumsub, P.; Kamchonwongpaisan, S.; Vanichtanankul, J.; Sirawaraporn, W.; Taylor, P.; Walkinshaw, M.D.; Yuthavong, Y. Insights into antifolate resistance from malarial DHFR-TS structures. Nat. Struct. Mol. Biol 2003, 10, 357-365.

20. Vanichtanankul, J.; Taweechai, S.; Yuvaniyama, J.; Vilaivan, T.; Chitnumsub, P.; Kamchonwongpaisan, S.; Yuthavong, Y. Trypanosomal dihydrofolate reductase reveals natural antifolate resistance. Chem. Biol 2011, 6, 905-911

21. Sanner, M. Python: A Programming Language for Software Integration and Development. J Mol Graphics Mod 1999,17, 57-61.

22. Morris, G.M.; Huey, R.; Lindstrom, W.; Sanner, M.F.; Belew, R.K.; Goodsell, D.S.; Olson, A.J. Autodock4 and AutoDockTools4: automated docking with selective receptor flexibility. J. Comp Chem 2009, 16, 2785-91

23. Huey, R.; Morris, G.; Forli, S. Using AutoDock 4 and AutoDock Vina with AutoDockTools: A Tutorial. The Scripps Research Institute Molecular Graphics Laboratory. California, USA. 2102.

24. Kim, S.; Chen, J.; Cheng, T.; Gindulyte, A.; He, J.; He, S.; Li, Q.; Shoemaker, B. A.; Thiessen, P. A.; Yu, B.; Zaslavsky, L.; Zhang, J.; Bolton, E.E. PubChem in 2021: new data content and improved web interfaces. Nucleic acids research 2019, 49(D1), D1388D1395.

25. Morris, G.M.; Huey, R.; Lindstrom, W.; Sanner, M.F.; Belew, R.K.; Goodsell, D.S.; Olson, A.J. Autodock4 and AutoDockTools4: automated docking with selective receptor flexibility. J. Comp Chem 2009, 16, 2785-91.

26. Forli, S.; Huey, R.; Pique, M.E.; Sanner, M.F.; Goodsell, D.S.; Olson, A. J. Computational protein-ligand docking and virtual drug screening with the AutoDock suite. Nature Protocols 2016, 115, 905-919. 
27. Pettersen, E.F.; Goddard, T.D.; Huang, C.C.; Couch, G.S.; Greenblatt, D.M.; Meng, E.C.; Ferrin, T.E. UCSF Chimera-A visualization system for exploratory research and analysis. J Comput Chem 2004, 25, 1605-1612.

28. Trott, O.; Olson, A.J.; AutoDock Vina: Improving the speed and accuracy of docking with a new scoring function, efficient optimization, and multithreading. J. Comput. Chem 2009, $31,2,455-461$.

29. Zali, S.; Mostoufi, A.; Afshoon, F.; Fereidoonnezhad, M. Design, molecular modelingand docking studies of novel Tacrine -aryl hybrids as multifunctional agents for the treatment of Alzheimer's disease. Int. J. Curr. Res. Chem. Pharm. Sci 2016, 3, 3, 71-72.

30. Lipinski, C.A.; Lombardo, F.; Dominy, B.W.; Feeney P.J. (March 2001). "Experimental and computational approaches to estimate solubility and permeability in drug discovery and development settings". Adv. Drug Deliv. Rev 2001, 46, 1-3, 3-26.

31. OSIRIS Property Explorer. Organic Chemistry Portal. Available at http://www.organicchemistry.org/prog/peo/. Accessed November 5, 2020

32. Humphrey, W.; Dalke, A.; Schulten, K. VMD: visual molecular dynamics. Journal of molecular graphics 1996, 14, 1, 33-8. 